\title{
Comparative Study of Rectangular, Trapezoidal and Parabolic Shaped Finned Heat sink
}

\author{
Ambeprasad.S.Kushwaha ${ }^{1}$, Prof. Ravindra Kirar $^{2}$ \\ ${ }^{1,2}$ (Mechanical, P.C.S.T, Bhopal/ R.G.P.V, India)
}

\begin{abstract}
This paper deals with the comparative study of heat sink having fins of various profiles namely Rectangular, Trapezoidal and Parabolic as heat sinks are the commonly used devices for enhancing heat transfer in electronic components. For the purpose of study heat sink is modeled by using the optimal geometric parameter such as fin height, fin thickness, base height, fin pitch as $48 \mathrm{~mm}, 1.6 \mathrm{~mm}, 8 \mathrm{~mm}, 2 \mathrm{~mm}$ and after that simulation is done at different heat load of 50W, 75W, 100W and with a air flow at 15CFM and air inlet temperature is taken as $295 \mathrm{~K}$. The simulation is carried out with a commercial package provided by fluent incorporation. The result obtained taking into consideration only the thermal performance.

As per the current era of the technological development everything is needed to be compact; whether it is a normal computer or laptop or the rack server we need everything that can be placed in a small space, so here the space constraint plays an major role as you cannot install a large heat sink for your device as it increases the size and the cost. So in this paper the pitch of fin is kept $2 \mathrm{~mm}$.
\end{abstract}

Keywords - Fin pitch, Heat Sink, Surface Nusselt Number, Temperature, Thermal Resistance.

\section{INTRODUCTION}

Extended surfaces or fins are commonly found on electronic components ranging from power supplies to transformers. The dissipation and subsequent rejection of potentially destructive self-produced heat is an important aspect of electronic equipment design. The dissipation of heat is necessary for its proper function. The heat is generated by the resistance encountered by electric current. Unless proper cooling arrangement is designed, the operating temperature exceeds permissible limit. As a consequence, chances of failure get increased.

In order to design an effective heat sink, some criterions such as a large heat transfer rate, a low pressure drop, high heat transfer coefficient, lowest maximum temperature attained, high surface Nusselt number, low thermal resistance, an easier manufacturing, a simpler structure, a reasonable cost and so on should be considered. The optimal geometric parameter is as per [3]. And the material of the construction is taken aluminum [10] as Aluminum has a thermal conductivity of 235 watts per Kelvin per meter (W/MK). (The thermal conductivity number, in this case 235 , refers to the metal's ability to conduct heat. Simply put, the higher the thermal conductivity number of a metal, the more heat that metal can conduct.) Aluminum is also cheap to produce and is lightweight. When a heat sink is attached, its weight puts a certain level of stress on the motherboard, which the motherboard is designed to accommodate. Yet the lightweight make up of aluminum is beneficial because it adds little weight and stress to the motherboard.

\section{OBJECTIVE OF WORK}

The main objective of the current work is:

2) To simulate the heat sink of the different fin profile for different heat input.

3) To predict temperature distribution along the channel.

4) To predict velocity, and temperature profiles for heat input of $50 \mathrm{w}, 75 \mathrm{w}, 100 \mathrm{w}$, applied on the base of the heat sink.

5) To define average heat transfer coefficient, surface Nusselt number and Maximum temperature attained, Thermal Resistance for the heat sink of the different fin profile for the inlet velocity of 15CFM and for heat input of 50w, 75w, 100w.

\section{COMPUTATIONAL SOFTWARE}

This section describes the fluent software required for carrying out a simulation and the process that are required to solve a problem using CFD. There is a variety of commercial CFD software available such as Fluent, Ansys CFX, ACE, as well as a wide range of suitable hardware and associated costs, depending on the complexity of the mesh and size of the calculations. 


\subsection{PROBLEMS-SOLVING WITH FLUENT}

There are many decisions to be made before setting up the problem in the fluent software. Some of the decisions to be made can include whether the problem should be 2D or 3D, which type of boundary conditions to use, whether or not to calculate pressure/temperature variations based on the air flow density, which turbulence model to use, etc. The assumptions made should be reduced to a geometry as simple as possible but retaining the most important features of the problem to be solved in order to reach close to an accurate solution.

After the above decisions are made, the geometry and mesh can be created. The grid should be made as fine as required to make the simulation grid independent. To determine the fineness required, a grid dependence study is normally carried out by making a series of refinements on an initially course grid, and carrying out simulations on each to determine when the key results of interest do not change, at which point the grid is considered independent

\subsection{STEPS IN CFD CALCULATION}

CFD codes are structured around the numerical algorithms that can be tackle fluid problems. In order to provide easy access to their solving power all commercial CFD packages include sophisticated user interfaces input problem parameters and to examine the results. Hence all Codes contain three main elements: In CFD calculations, there are three main steps

1. Pre-processing.

2. Solver

3. Post processing.

Pre-Processing is the step where the modeling goals are determined and computational grid is created. In the second step numerical models and boundary conditions are set to start up the solver. Solver runs until the convergence is reached. When solver is terminated, the results are examined which is the post processing part.

PRE-PROCESSING: Pre-Processing is the step where the modeling goals are determined and computational grid is created. Preprocessor consists of input of a flow problem by means of an operator friendly interface and subsequent transformation of this input into form of suitable for the use by the solver.

Definition of fluid properties: Specification of appropriate boundary conditions at cells, which coincide with or touch the boundary. The solution of a flow problem (velocity, pressure, temperature etc.) is defined at nodes inside each cell. The accuracy of CFD solutions is governed by number of cells in the grid. In general, the larger numbers of cells better the solution accuracy. Both the accuracy of the solution \& its cost in terms of necessary computer hardware \& calculation time are dependent on the fineness of the grid. Efforts are underway to develop CFD codes with a (self) adaptive meshing capability. Ultimately such programs will automatically refine the grid in areas of rapid variation.

SOLVER EXECUTION: In the second step numerical models and boundary conditions are set to start up the solver. Solver runs until the convergence is reached. There are three distinct streams of numerical solutions techniques: finite difference, finite volume\& finite element methods. In outline the numerical methods that form the basis of solver performs the following step the approximation of unknown flow variables are by means of simple functions. Discretization by substitution of the approximation into the governing flow equations \&subsequent mathematical manipulations

POST-PROCESSING: As in the pre-processing huge amount of development work has Recently has taken place in the post processing field. Owing to increased popularity of engineering work stations, many of which has outstanding graphics capabilities, the leading CFD

Are now equipped with versatile data visualization tools.

- Domain geometry and grid display

- Vector plots

- Line and shaded contour plots

- $2 \mathrm{D}$ and 3D surface plots

- Particle tracking

- Animation for dynamic result

\subsection{CFD GOVERNING EQUATIONS SOLVE BY FLUENT}

This section is a summary of the governing equations used in CFD to mathematically solve for fluid flow and heat transfer, based on the principles of conservation of mass, momentum, and energy. These equations solve by the fluent software.

The conservation laws of physics form the basis for fluid flow governing equations. 
Law of Conservation of Mass:

$$
\frac{\partial \rho}{\partial t}+\nabla \cdot(\rho \vec{V})=0
$$

Momentum Equation:

X-momentum:

$\frac{\partial(\rho u)}{\partial t}+\nabla \cdot(\rho u \vec{V})=-\frac{\partial p}{\partial x}+\frac{\partial \tau_{x x}}{\partial x}+\frac{\partial \tau_{y x}}{\partial y}+\frac{\partial \tau_{z x}}{\partial z}+S_{M x}$

Y-momentum:

$\frac{\partial(\rho v)}{\partial t}+\nabla \cdot(\rho v \vec{V})=-\frac{\partial p}{\partial y}+\frac{\partial \tau_{x y}}{\partial x}+\frac{\partial \tau_{y y}}{\partial y}+\frac{\partial \tau_{z y}}{\partial z}+S_{M y}$

Z-momentum:

$$
\frac{\partial(\rho w)}{\partial t}+\nabla \cdot(\rho w \vec{V})=-\frac{\partial p}{\partial z}+\frac{\partial \tau_{x z}}{\partial x}+\frac{\partial \tau_{y z}}{\partial y}+\frac{\partial \tau_{z z}}{\partial z}+S_{M z}
$$

Energy Equation:

$$
\frac{\partial\left(\rho h_{0}\right)}{\partial t}+\nabla \cdot\left(\rho h_{0} \vec{V}\right)=-p \nabla \cdot \vec{V}+\nabla \cdot(k \nabla T)+\Phi+S_{h}
$$

Equation of state:

$$
p=\rho R T
$$

Where $\rho$ is the density, $\mathrm{u}, \mathrm{v}$ and $\mathrm{w}$ are velocity components, $\mathrm{V}$ is the velocity vector, $\mathrm{p}$ is the pressure, $\mathrm{S}$ terms are the source terms and terms are the viscous stress components which are defined for a Newtonian fluid as.

$$
\begin{gathered}
\tau_{x x}=\lambda\left(\frac{\partial u}{\partial x}+\frac{\partial v}{\partial y}+\frac{\partial w}{\partial z}\right)+2 \mu \frac{\partial u}{\partial x} \\
\tau_{y y}=\lambda\left(\frac{\partial u}{\partial x}+\frac{\partial v}{\partial y}+\frac{\partial w}{\partial z}\right)+2 \mu \frac{\partial v}{\partial y} \\
\tau_{z z}=\lambda\left(\frac{\partial u}{\partial x}+\frac{\partial v}{\partial y}+\frac{\partial w}{\partial z}\right)+2 \mu \frac{\partial w}{\partial z} \\
\tau_{x y}=\tau_{y x}=\mu\left(\frac{\partial u}{\partial y}+\frac{\partial v}{\partial x}\right)
\end{gathered}
$$




$$
\begin{aligned}
& \tau_{x z}=\tau_{z x}=\mu\left(\frac{\partial u}{\partial z}+\frac{\partial w}{\partial x}\right) \\
& \tau_{y z}=\tau_{z y}=\mu\left(\frac{\partial v}{\partial z}+\frac{\partial w}{\partial y}\right)
\end{aligned}
$$

\section{MODELING AND ANALYSIS}

The procedure for solving the problem is:

- Create the geometry.

- Mesh the domain.

- Set the material properties and boundary conditions.

- Obtaining the solution

Modeling software UNI-Graphics NX-6 creates the geometry and the geometry is imported to the Ansys workbench 14.0 where meshing is done, and exports the mesh to FLUENT. The boundary conditions, material properties, and surrounding properties are set through parameterized case files. FLUENT solves the problem until either the convergence limit is met, or the number of iterations specified by the user is achieved [11].

\subsection{PREPARATION OF THE CAD MODEL}

The Sketching is done as per dimensions of the heat sink as suggested in [3]. After this process the constraint are applied and this way the model is achieved in modeling software UNI-Graphics NX-6.

\subsection{MESHING OF THE DOMAIN}

The second part of pre-processing is the mesh generation. After the model is imported to Ansys workbench it is then launched in the meshing module for the mesh generation Coarse, medium, and fine mesh types are available. Mesh density varies based upon the assigned Refinement Factor. Mesh is the key component of a high quality solution. There are three kinds of meshing algorithms. These are Hexahedral Cartesian, Hexahedral Unstructured and Tetrahedral meshers. Hexahedral Cartesian mesher is the one generating fully structured meshes. It is suitable for very limited type of geometries. It is generally inappropriate for models where curved surfaces exist. Hexahedral Unstructured mesher creates grids of hexahedral cells dominantly and tetrahedral cells where necessary. Tetrahedral mesher is designed for very complicated geometries where the other two cannot be used. For models involving spheres or ellipsoids hexahedral meshers are useless. In our problem CFD unstructured mesher is used.

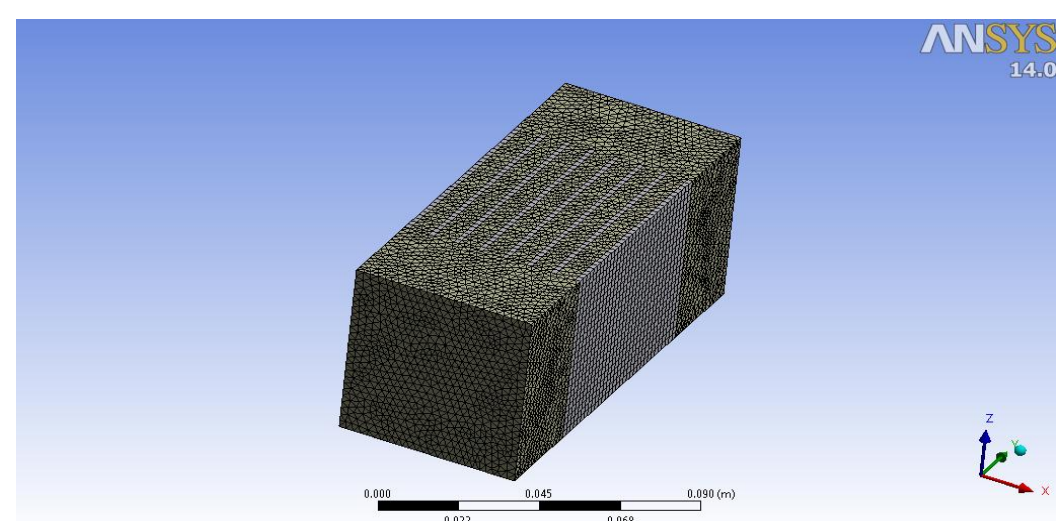

Fig. 4.1 Meshing of Whole domain

\subsubsection{NECESSITY OF MESH REFINEMENT}

The primary benefit of these techniques is their increased efficiency that results from meshing, i.e. from the improvement of the existing mesh which more efficiently represents the pertinent parts of the problem. 
In numerical analysis, mesh refinement is a method of meshing. Central to any Eulerian method is the manner in which it discretises the continuous domain of interest into a grid of many individual elements. This grid may be static, established once and for all at the beginning of the computation, or it may be dynamic, tracking the features of the result as the computation progresses. If the computation has features which one wants to track which are much smaller than the overall scale of the problem, and which move in time, then one must either include many more static grids to cover the region of interest, or adopt a dynamic scheme.

\section{TABLE. 1 RESUlT}

\begin{tabular}{|c|c|c|c|c|c|c|}
\hline Fin Profile & Fin pitch & $\begin{array}{l}\text { Heat } \\
\text { input }\end{array}$ & $\begin{array}{c}\text { overall heat } \\
\text { transfer } \\
\text { coefficient } \\
\text { W/M2 K }\end{array}$ & $\begin{array}{c}\text { Max } \\
\text { temperature } \mathrm{K}\end{array}$ & $\begin{array}{l}\text { Nusselt } \\
\text { Number }\end{array}$ & $\begin{array}{c}\text { Thermal } \\
\text { resistance } \\
\text { K/W }\end{array}$ \\
\hline \multirow{3}{*}{ Rectangular } & \multirow{3}{*}{$2 \mathrm{~mm}$} & $50 \mathrm{~W}$ & 30.50709 & 325.8217 & 1260.624 & 0.6161 \\
\hline & & $75 W$ & 32.84953 & 341.2246 & 1357.418 & 0.6163 \\
\hline & & $100 \mathrm{~W}$ & 34.16299 & 356.636 & 1411.694 & 0.6163 \\
\hline \multirow{3}{*}{ Trapezoidal } & \multirow{3}{*}{$2 \mathrm{~mm}$} & $50 \mathrm{~W}$ & 36.32706 & 325.1658 & 1501.118 & 0.6033 \\
\hline & & $75 W$ & 38.9974 & 340.2493 & 1611.463 & 0.6033 \\
\hline & & $100 \mathrm{~W}$ & 40.47094 & 355.1844 & 1672.353 & 0.6011 \\
\hline \multirow{3}{*}{ Parabolic } & \multirow{3}{*}{$2 \mathrm{~mm}$} & $50 W$ & 33.38483 & 328.7138 & 1379.538 & 0.6741 \\
\hline & & $75 W$ & 35.61325 & 345.56387 & 1471.622 & 0.6741 \\
\hline & & $100 \mathrm{~W}$ & 36.84299 & 362.42621 & 1522.438 & 0.6741 \\
\hline
\end{tabular}

\subsection{RESULTS \&CONTOURS}

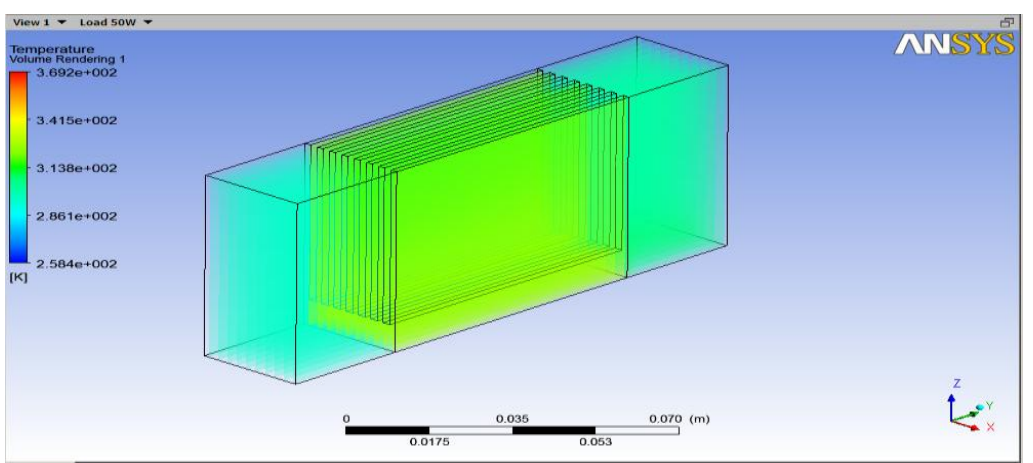

Fig 4.2 Contour of Static Temperature of $2 \mathrm{~mm}$ Fin pitch Rectangular Heat sink with Heat input of 50W

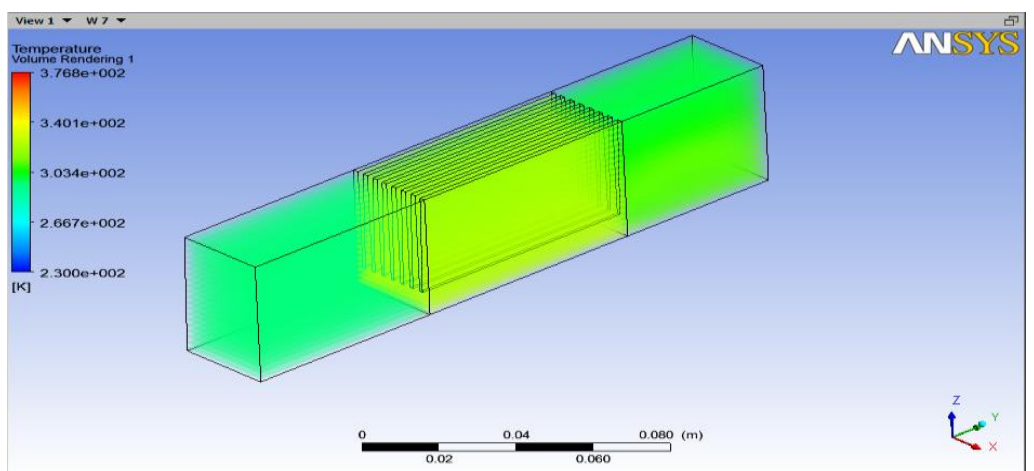

Fig.4.3 Contour of Static Temperature of $2 \mathrm{~mm}$ Trapezoidal Heat sink with Heat input of 50W 


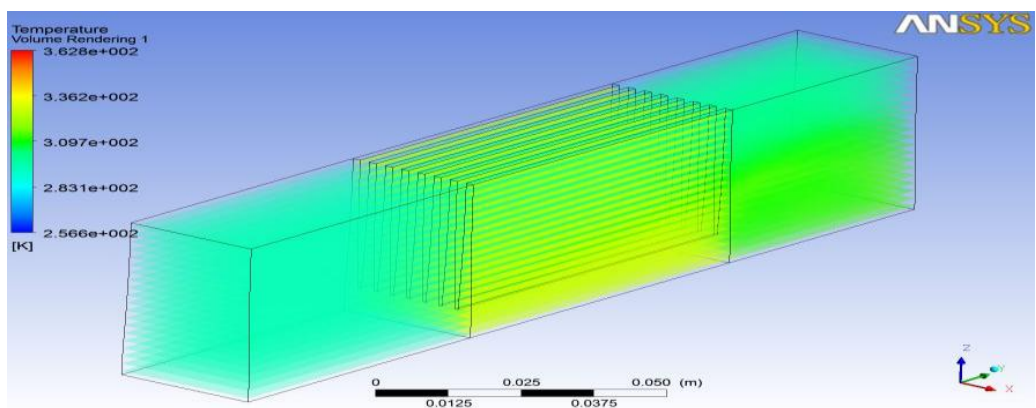

Fig.4.4 Contour of static Temperature of 2mm Fin pitch Parabolic Heat sink with Heat input of 50W

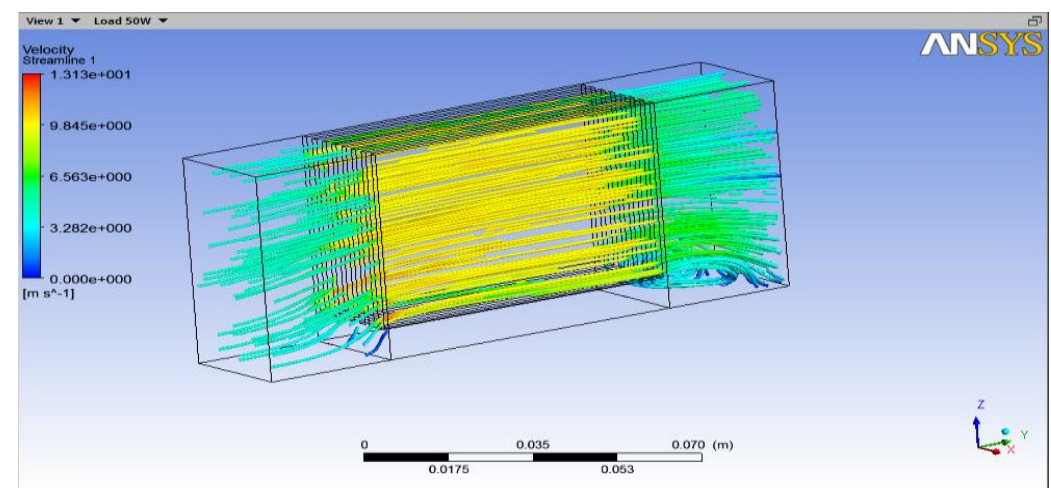

Fig.4.5 Velocity contour of 2mm Fin pitch Rectangular Heat sink with Heat input of 50W

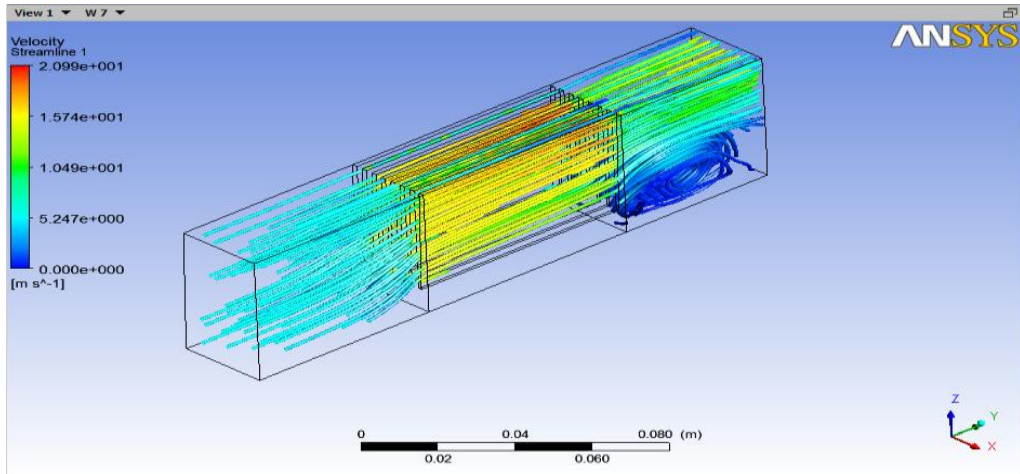

Fig.4.6 Velocity contour of 2mm Fin pitch Trapezoidal Heat sink with Heat input of 50W

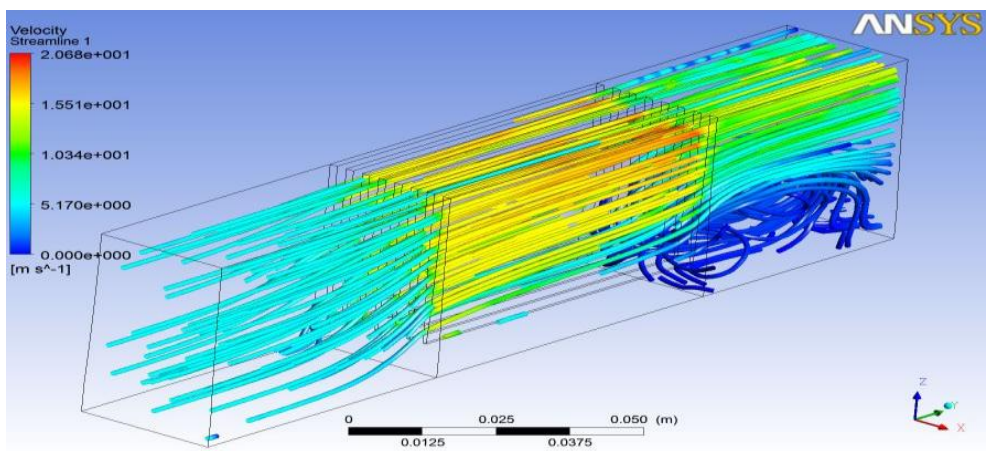

Fig.4.7 Velocity contour of 2mm Fin pitch Parabolic Heat sink with Heat input of 50W

\section{CONCLUSION}

For all the three profile compared for the maximum temperature attained on the basis of result governed by the CFD analysis, with the fin pitch $2 \mathrm{~mm}$ the Trapezoidal heat sink shows that the maximum temperature attained is minimum as compared to the Rectangular and Parabolic heat sink and the parabolic heat sink shows the highest maximum temperature attained which is not desirable. 
Heat transfer coefficient and surface Nusselt number is Maximum in Trapezoidal heat sink for all the fin pitch and for different heat loads of $50 \mathrm{~W}, 75 \mathrm{~W}, 100 \mathrm{~W}$.

As per the criterion for the selection of heat sink, the heat sink should have lowest thermal resistance and maximum heat transfer coefficient. The Trapezoidal Heat sink shows the lowest thermal resistance and the maximum heat transfer coefficient.

From the calculated values we can find that the best configuration for this type of convective heat transfer of a heated sink is a heat sink with TRAPEZOIDAL fin with a fin pitch of $2 \mathrm{~mm}$ as they have the highest total heat transfer rate as it has the lowest maximum temperature attained compared to other, lowest thermal resistance best surface Nusselt number along with highest surface heat transfer coefficient for a given heat load of 50W, $75 \mathrm{~W}, 100 \mathrm{~W}$.

\section{REFERENCES}

[1]. Behnia M, \& Copeland D, and Sood phadakee D.1998, "A Comparison of Heat Sink Geometries for Laminar Forced Convection," Proceedings of The Sixth Intersociety Conference on Thermal and Thermo mechanical Phenomenon Electronic Systems, Seattle, Washington, USA, May 27-30, pp. 310-315.

[2]. Mohsin et al. "Optimization of Cylindrical Pin-fin heat sinks using Genetic Algorithm" IEEE Transactions on components and packaging technologies, Vol. 32, No. 1, March 2009.

[3]. Arularasan R. and Velraj R. "CFD Analysis in a Heat sink for cooling of Electronic devices" International Journal of The Computer, the Internet and Management Vol. 16.No.3 (September-December, 2008) pp 1-11

[4]. Christopher L \& Chapman, Seri Lee, Bill L. Schmidt. "Thermal Performance of an Elliptical Pin Fin Heat Sink," Tenth IEEE SEMITHERM, pp.24-31, 1993.

[5]. W. A. Khan, J. \& R. Culham \& M. M. Yovanovich. "The Role of Fin Geometry in Heat Sink Performance" Transactions of the ASME Vol. 128, dec 2006.

[6]. Laxmidhar Biswal \& Suman Chakraborty and S. K. Som. "Design and Optimization of Single-Phase Liquid Cooled Microchannel Heat Sink" IEEE Transactions on components and packaging technologies, Vol. 32, No. 4, December 2009.

[7]. Vafai, K. and Zhu, Lu., (1999), "Analysis of two-layered micro channel heat sink concept in electronic cooling", International Journal of Heat and Mass Transfer, Vol. 42, pp. 2287-2297.

[8]. Shashank Deorah "CFD Analysis of a vertical tube having internal fins for the Natural Convection"

[9]. Kai shing yang \& yur tsai lin "Heat transfer characteristic of heat sink influence of fin spacing at low Reynolds number region" International journal of Heat and Mass Transfer vol.53 issue 25-26 Dec 2010

[10]. Emre Ozturk and Ilker Tari "Forced Air Cooling of CPUs with Heat Sinks: A Numerical Study" IEEE transactions on components and packaging technologies, Vol. 31, NO. 3, Sep 2008

[11]. Heat transfer through fins "Fluent incorporation [flow lab 1.2]" April 12, 2007. 